\title{
Pericentric inversion (13) with two different recombinants in the same family
}

\author{
E M WILliaMSON,* J F MILLER, $\dagger$ AND M SEABRIGHT
}

From *the Department of Child Health, and the Department of Human Reproduction and Obstetrics, University of Southampton, Southampton; and $\ddagger$ the Wessex Regional Cytogenetics Unit, Salisbury, Wiltshire

SUMMARY A family is described in which a pericentric inversion (13) was discovered in the father after the birth of an abnormal baby. In a further pregnancy amniocentesis was carried out. The fetal karyotype showed a rec(13)dup p,inv(13)(p11q22). The fetus's abnormalities were similar to those observed in the first child. Family studies showed that a first cousin, mentally retarded, had a rec(13)dup q,inv(13)(p11q22) karyotype. In this family, the risk of occurrence of a recombinant in offspring of an inversion carrier could be as high as $40 \%$.

Several families have been described in which a pericentric inversion in a phenotypically normal parent has given rise to a duplication/deficiency recombinant in a phenotypically abnormal offspring. ${ }^{1-3} \mathrm{We}$ report here a family in which a pericentric inversion of a chromosome 13 was found after the birth of an abnormal infant. Within this family two types of Received for publication 17 December 1979 recombinant were discovered causing different sets of abnormalities.

\section{Family report (fig 1)}

The parents (III.5 and III.6) were aged 30 and 28 years, respectively, when their first child (IV.1) was born at term in 1970. Pregnancy was complicated by

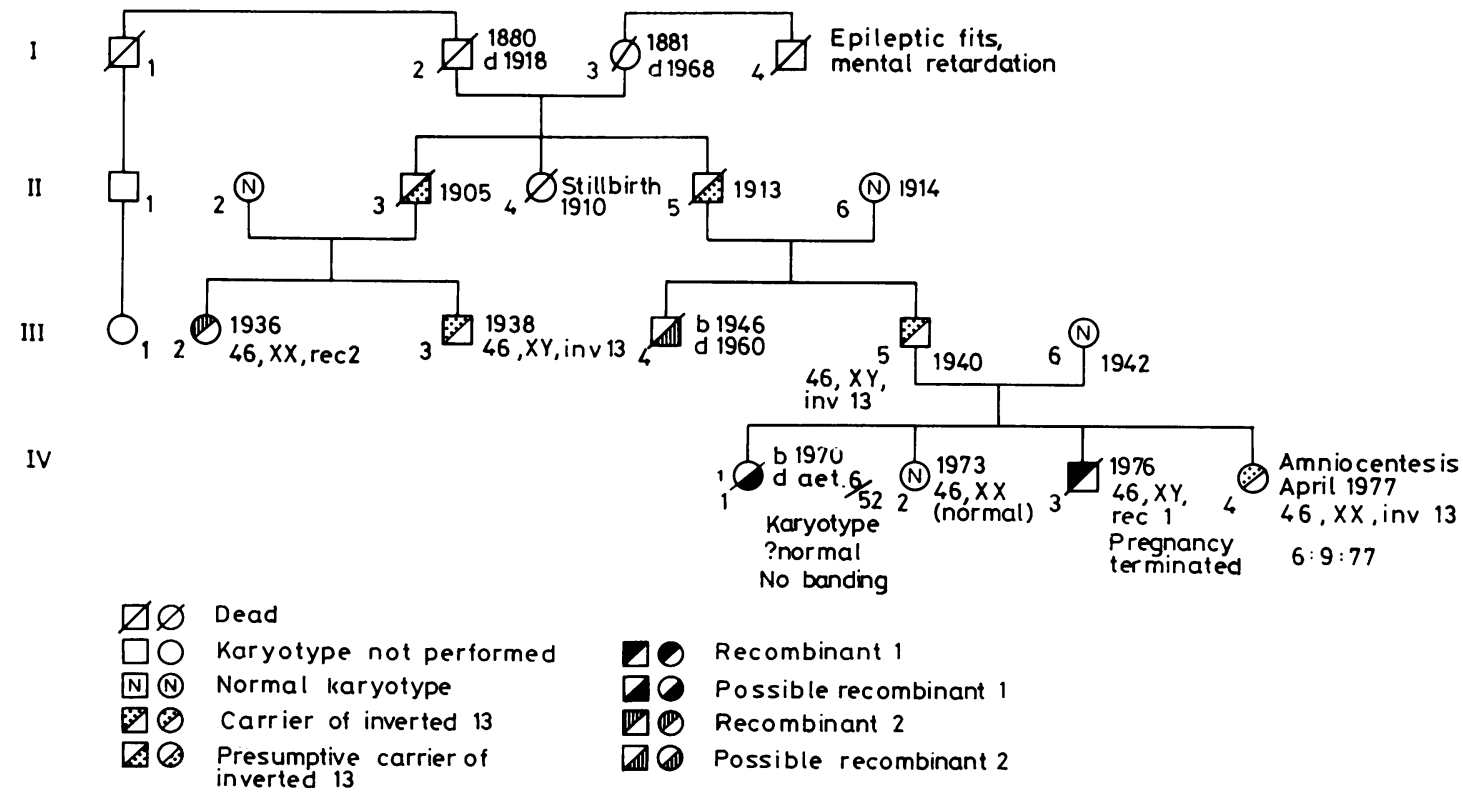

FIG 1 Family pedigree 


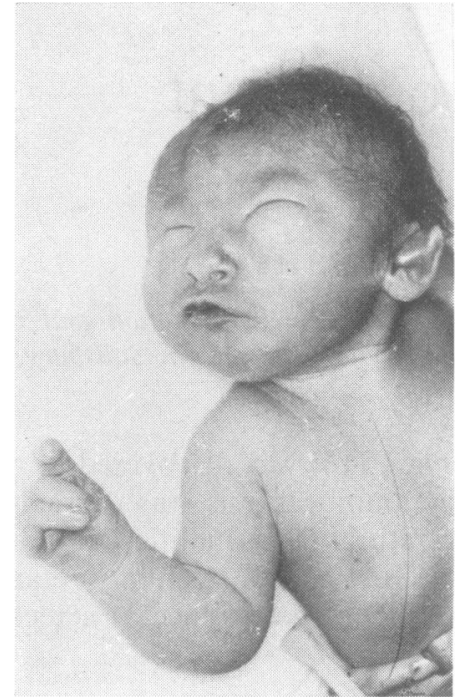

FIG 2 IV.1 showing microphthalmia, abnormal ears, and absent thumb.

a threatened abortion at 3 months but was otherwise normal. The $1600 \mathrm{~g}$ female infant had multiple congenital abnormalities (fig 2). These were microcephaly, severe bilateral microphthalmia, low set abnormal ears, bilateral absent thumbs with long slender fingers, and an imperforate anus with a fistula near the vaginal orifice. She failed to thrive and died at the age of 6 weeks from bronchopneumonia, her weight at that time being only $1080 \mathrm{~g}$. In addition to the external anomalies, necropsy examination showed a very small brain $(140 \mathrm{~g})$ with all its lobes reduced in size, particularly the frontal lobes. No other abnormality was noted, but a dislocation of both hip joints was suspected. Unbanded chromosome analysis appeared normal.

After the loss of this baby, the parents proceeded with another pregnancy in 1973, which resulted in the birth of a normal female infant (IV.2).

The parents sought genetic counselling in 1974. At this time it was felt that the abnormalities in the first baby could have been chromosomal in origin, so karyotypes of both parents and the surviving child were prepared using banding techniques. The mother and child (III.6 and IV.2) were normal $(46, X X)$, but it was found that the father (III.5) carried a pericentric inversion of a chromosome 13, 46,XY,inv(13)(p11q22) (fig 3a). Amniocentesis for fetal karyotyping was advised in any future pregnancy because of the risk of a recombinant.

The mother conceived again in 1976. Amniocentesis was carried out at 16 weeks and the fetal
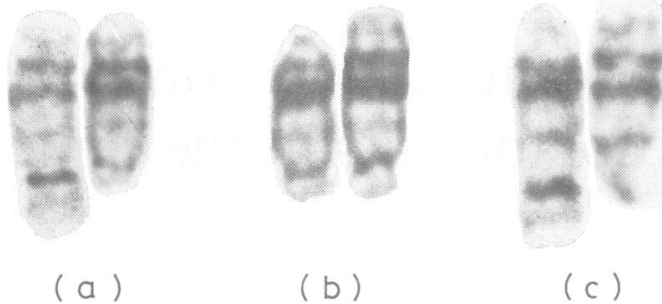

(c)

FIG 3 (a) Pericentric inversion (13) present in the father (III.5); (b) recombinant 1 (IV.3); (c) recombinant $2 \overrightarrow{\vec{\omega}}$ (III.2).

karyotype showed a recombinant of the inverted $13 . \overrightarrow{.}$ with duplication of the short arm and deficiency of $f_{\dot{\omega}}^{+}$ the long arm 46,XY,rec(13)dup p,inv(13)(p11q22)응 (fig 3b). The pregnancy was terminated and the maleo fetus (IV.3) was found to have microcephaly, microphthalmia, and absent thumbs. These were similar to the abnormalities found in the first child (fig 4) Sterilisation was offered to the mother at the time of the termination of pregnancy but the parents_ wished to postpone such an irreversible decision ${ }_{\infty}$ until there had been a reassessment of the genetic. risk with further family data.

Chromosome analysis of the father's paternals first cousins was performed. The normal male (III.3) was found to carry the pericentric inversion. His elder sister (III.2) had always been considered $\stackrel{\circ}{\circ}$

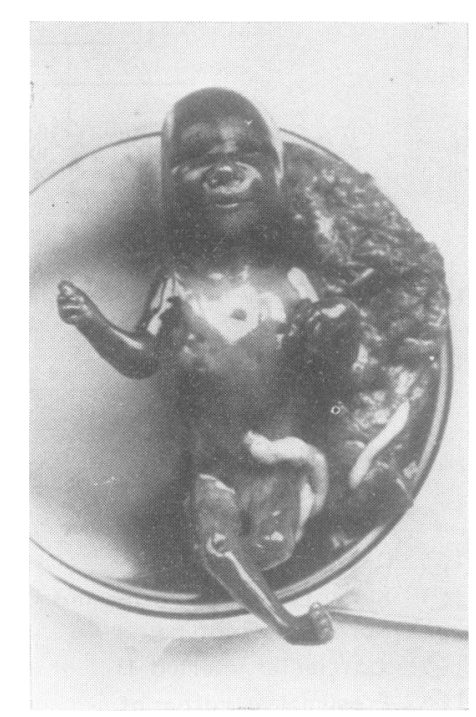

FIG 4 Terminated fetus (IV.3) showing features similar to IV.I. 
to have Down's syndrome, but chromosome analysis had never been performed. She was short $(149 \mathrm{~cm})$ and mentally retarded with ptosis, cleft soft palate, and polydactyly. There was a history of fits during childhood. Her karyotype showed a second recombinant of the inverted 13 with duplication of part of the long arm 46,XX,rec(13)dup q,inv(13)(p11q22) (fig 3c). The father's only sib was a brother (III.4) who died at the age of 14 years. $\mathrm{He}$ was severely retarded and had polydactyly. He also had fits during childhood.

A high risk of recurrence of abnormality was given, and the couple decided that this was unacceptable and requested sterilisation. However, pregnancy had already occurred and amniocentesis was again performed at 16 weeks. This time the fetus was 46,XX,inv(13)(p11q22). The pregnancy continued to term and a phenotypically normal female infant was delıvered (IV.4).

\section{Discussion}

The estimation of genetic risk in this family had, in the first instance, to be based upon the clinical assessment of the abnormalities found in the first child. Microcephaly, microphthalmia, absent thumbs, imperforate anus, and congenital dislocation of the hips have been described as occurring together in partial 13q monosomy. ${ }^{4}$ When the father was found to carry an inversion of a chromosome 13 , it seemed justifiable to assume that this was contributory to the occurrence of abnormalities in the child, and that there was a risk of recurrence.

Sutherland et $a l^{5}$ pooled the available data from reported families in which inversions of various chromosomes had been ascertained by the birth of an abnormal baby, and from these figures they defined a risk of recurrence of abnormality of $10 \%$ if the female parent carried the inversion and $5 \%$ if the male parent were the carrier. The authors accept that grouping together this heterogeneous data might not be reliable, but it could provide a basis for genetic counselling.

In a review of 33 amniocenteses carried out because of parental inversions, Boué (1978, personal communication) found that $\mathbf{1 7}$ of the fetuses carried the inversion, 13 had normal karyotypes, and three recombinants were detected. In this group the risk of a recombinant in the offspring of an inversion carrier is in broad agreement with Sutherland's figure of $10 \%$, and this was the risk given to the parents (III.5 and 6).

After amniocentesis, the finding of microphthalmia, microcephaly, and absent thumbs in the terminated fetus carrying a recombinant confirmed our assumption that these abnormalities were associated with chromosomal imbalance. When a second viable recombinant was found with the much less specific abnormalities of mental retardation, polydactyly, and cleft palate, known to be associated in partial trisomy 13, a reassessment of genetic risk was made.

Warter $e t a l^{6}$ reported a family in which a father carrying a pericentric inversion of chromosome 5 had two abnormal offspring, each carrying a different recombinant. They estimated the risk of recurrence of abnormality to be 2 in 6 in their family. It would seem that the risk must depend upon the chromosome involved, the size and position of the inversion, and the viability of recombinants, and thus each family must be considered individually.

In each of the three previously described families with inversions of a chromosome $13,{ }^{1-3}$ only one viable recombinant was reported, but in each family there was a history of spontaneous abortions which could represent intrauterine death of recombinants. Taysi et al, ${ }^{3}$ recognising that both recombinants were potentially viable in their family, estimated the risk of abnormality to be 1 in 5 .

In the family reported here there were no spontaneous abortions noted and both recombinants were viable. The findings of two recombinants among the six karyotyped subjects (offspring of obligatory inversion carriers) gives an occurrence rate of 1 in 3, and if those whose chromosomes were not examined are included, four in ten subjects were abnormal. In all cases the inversion was transmitted through male parents and a risk of $40 \%$ can be attributed to offspring of male carriers. The empirical risk to offspring of female carriers cannot be estimated from the family data, but the risk must be high. This is an obvious indication for amniocentesis and fetal karyotyping in any future pregnancy.

We would like to thank Dr P A Manfield, Consultant Paediatrician, Good Hope General Hospital, Sutton Coldfield, for details and photograph of case IV.1, and Mr Peter Payne, Consultant Obstetrician at Basingstoke District Hospital, who performed the termination.

\footnotetext{
References

1 Parrington JM, Edwards JH. Patau's syndrome with D duplication-deficiency derived from a maternal D group pericentric inversion. Ann Hum Genet 1971;35:35-45.

2 Hauksdottir H, Halldorsson S, Jensson O, Mikkelsen M, McDermott A. Pericentric inversion of chromosome No 13 in a large family leading to duplication deficiency causing congenital malformations in three individuals. $J$ Med Genet 1972;9:413-21.
} 
3 Taysi K, Bobrow M, Balci S, Madan K, Atasu M, Say B. Duplication/deficiency product of a pericentric inversion in man: a cause of $D_{1}$ trisomy syndrome. J Pediatr 1973; 82:263-8.

4 de Grouchy J, Turleau C. Clinical atlas of human chromosomes. New York: Wiley, 1977:135.

5 Sutherland GR, Gardiner AJ, Carter RF. Familial pericentric inversion of chromosome 19, inv (19)(p13q13) with a note on genetic counselling of pericentric inversion carriers. Clin Genet 1976;10:54-9.
6 Warter S, Ruch JV, Lehmann M. Karyotype with chromo-六 somal abnormality with various inherited defects in the $\mathbb{D}_{+}$ offspring (recombination aneusomy). Hum Genet 1973; 20:355-9.

Requests for reprints to Dr E M Williamson, Department of Child Health, Southampton General Hospital, Shirley, Southampton SO9 4XY. 